\title{
Thermodynamic Limits of Work and Pressure Gain in Combustion and Evaporation Processes
}

\author{
M. Razi Nalim* \\ Indiana University-Purdue University Indianapolis, Indianapolis, Indiana 46202
}

\begin{abstract}
Combustion and evaporation processes occurring in a closed chamber can result in significant pressure rise and direct work transfer. The pressure and volumetric changes that accompany such processes allow substantial work potential to be achieved in cyclic nonsteady devices, such as internal combustion engines and pulsed combustion or detonation engines. The ideal pressure gain or work production is a function of the prescribed inflow and outflow conditions, volumetric confinement, fluid properties, and other parameters. The generalized thermodynamic limits of pressure gain and work production in such devices are investigated. Analytic and iterative methods are provided to evaluate cyclic combustion and evaporation processes for enhancing airbreathing combustion engine performance.
\end{abstract}

$\begin{aligned} c & \\ c & =\text { Nomecific heat } \\ h & =\text { specific enthalpy } \\ I & =\text { impulse } \\ m & =\text { mass } \\ p & =\text { pressure } \\ Q & =\text { sensible heat release of combustion } \\ Q^{\prime} & =\text { latent heat of evaporation } \\ R & =\text { mass-specific gas constant } \\ T & =\text { temperature } \\ u & =\text { specific internal energy } \\ v & =\text { specific volume } \\ W & =\text { work } \\ \alpha & =\text { moles of injected water } \\ \gamma & =\text { specific heat ratio, } c_{p} / c_{v} \\ \eta & =\text { adiabatic efficiency } \\ \mu & =\text { mass fraction } \\ \phi & =\text { fuel-air equivalence ratio }\end{aligned}$

\section{Subscripts}

$A=$ state after precompression
$A^{\prime}=$ state after evaporation
$B=$ state after combustion
$L=$ liquid, injection state
$p=$ constant pressure
$r=$ residual buffer gas
$s p=$ specific
$v=$ constant volume
$2=$ device inlet state or mass
$3=$ device outlet state or mass

\section{Introduction}

\begin{abstract}
ATHOUGH conventional internal combustion (IC) engines have long been used to develop power from an intermittent combustion process, there has been a recent growth in interest in other pulsed combustion devices such as pulsed detonation engines

Presented as Paper 98-3398 at the 34th Joint Propulsion Conference, Cleveland, OH, July 1998; received 26 July 2001; revision received 5 June 2002; accepted for publication 5 July 2002.

Copies of this paper may be made for personal or internal use, on condition that the copier pay the $\$ 10.00$ per-copy fee to the Copyright Clearance Center, Inc., 222 Rosewood Drive, Danvers, MA 01923; include the code $0748-4658 / 02 \$ 10.00$ in correspondence with the CCC.

*Assistant Professor, Mechanical Engineering Department, 723 West Michigan Street, Suite 260. Senior Member AIAA.
\end{abstract}

(PDE) and wave rotors, as well as alternative (desirably more efficient and less-polluting) IC engine designs. The nonsteady combustion chamber(s), either singly, or more commonly in multiples, can be connected to nominally steady flow devices such as inlets, compressors, nozzles, and turbines. Unlike piston engines, the work potential generated in such pulsed combustion engines is made available primarily as pressure waves and the kinetic energy of the gas exiting the combustion chamber.

These features prevent such systems from being analyzed using air-standard cycles that either assume a homogeneous controlmass closed system for piston engines (for example, Otto cycle) or assume steady-flow control-volume component systems for gas turbine engines (Brayton cycle). Figure 1 is a pressure-volume diagram for these familiar cycles, as indicated, with 1-2-3-4-1 being the "Humphrey cycle" that has been proposed ${ }^{1}$ for analyzing constantvolume pulsed combustion devices. In the Otto cycle $\left(1-2-3-4^{\prime}-1\right)$ the work done $\left(\int p \mathrm{~d} v\right)$ in each process of a closed system leads to a different geometric interpretation from that for the work done $\left(\int v \mathrm{~d} p\right)$ in each steady flow process of the open system components of a Brayton cycle (1-2-3'-4-1). Although the Humphrey and Otto cycles share a constant-volume heat addition process, the former achieves a complete expansion to inlet pressure, generally in a separate expansion device or during outflow, whereas the latter achieves only partial expansion (to the initial volume at inlet, but not the initial pressure) within the closed system. Even though actual piston engines might be open for inlet and exhaust, the air-standard Otto cycle considers a completely closed system.

Because pulsed combustion systems under consideration are neither closed nor steady, the Humphrey cycle (also called Atkinson cycle) could be misleading if either of the conventional geometric interpretations ${ }^{2}$ are made, or if end state 3 of the combustion process in Fig. 1 was misinterpreted as the inflow state of the downstream turbine or nozzle. Rather, it is necessary to account for flow work explicitly between the steady and nonsteady components. There appears to have been some confusion in early discussions of the potential of PDEs that can be attributed to the preceding difficulty. This study offers a method to connect the control-mass analysis of the process in a temporarily closed combustion chamber to the control-volume analysis required from the open system viewpoint. It leads to the establishment of thermodynamic limits on the benefits offered from pulsed combustion and evaporation processes.

A pressure-gain combustor is a device designed to produce an increase in pressure during combustion. Examples of such devices are pulse combustors, reciprocating engines, free piston combustors, wave rotors (also called dynamic pressure exchangers), and pulse detonation engines. ${ }^{3,4}$ These devices necessarily employ nonsteady, intermittent combustion, accomplishing an internal work transfer from burning gas to surrounding gas through pressure waves. The 


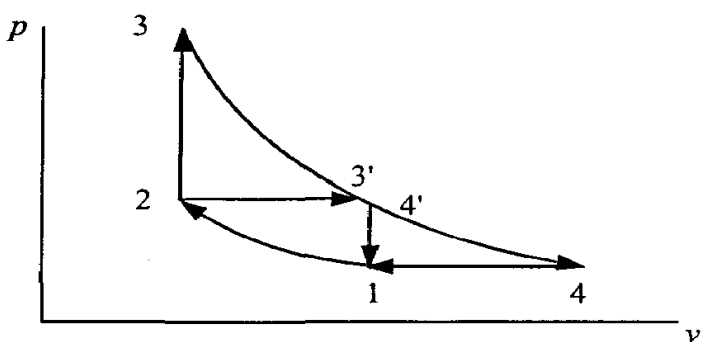

Fig. 1 Comparison of thermodynamic cycles.

pressurization of most gas prior to burning causes a decrease in entropy production, and this is at the heart of the thermodynamic benefit. It might be necessary to use a number of identical combustor units in parallel in order to present a nearly steady flow to other components.

In a gas turbine the allowable turbine temperature limits the temperature change across the combustor. In a direct thrust engine the exit velocity might be limited to improve propulsive efficiency or reduce noise. The energy release in the combustor is dictated by these limits, irrespective of the internal process. Although the pressure gain is determined primarily by the temperature limits, other controllable design parameters that influence the pressure gain significantly are the focus of this paper.

A work-producing combustor is a similar combustion device, designed instead to produce mechanical work by some means, with the gas exiting the device limited to a specified temperature and a specified pressure, which might be the same as the inlet pressure. Examples are a reciprocating engine and a wave turbine. ${ }^{3,5}$ An exit temperature limit might be imposed by a downstream turbine or by a stoichiometric combustion limit. Again, the ideal work is determined primarily by the energy addition dictated by these limits, but other design parameters must be optimized to maximize the work output.

The evaporation of a liquid fuel or other liquid in the combustor will contribute to work potential, as will the changes in the gas molecular weight and specific heat as a result of the chemical conversion and large temperature change. These effects might be significant and could be included by incorporating real-gas thermodynamic properties into a detailed numerical analysis. However, it is useful to separate the effects of changes in temperature and molar density, in order to understand these processes individually relative to air-standard analysis.

Here, evaporation is treated simply as an increase in the number of moles of gas. The heat of evaporation is subsumed (but not neglected) in the overall energy balance, which is dominated by combustion. The evaporation of fuel or another liquid boosts the pressure and work potential of the resulting gas, compared with the negligible flow work in introducing liquid due to very low specific volume. This effect is separate from the power boost available from the additional mass flow and the latent heat of the liquid allowing extra heat addition within a temperature limit.

Given a temperature limit, the ideal (potential) pressure gain or work output is determined here. The benefit obtained will be degraded by leakage, heat loss, and the inefficiencies of the charging and discharging aerodynamic processes in the particular nonsteady device used. Losses caused by motion and work exchange are represented here by adiabatic compression and expansion efficiencies, but heat loss and leakage are neglected. Ideal-gas thermodynamic analysis is used, assuming negligible kinetic energy in the combustion space. Although these assumptions apply plausibly to free-piston, wave-rotor, and pulse combustors, it is argued next that the results also apply to detonation devices. Further, the total combustor volume is assumed held unchanged during evaporation and combustion, which are normally rapid in relevant systems. However, there might be residual "buffer" gas, which allows the working gas experiencing combustion or evaporation to expand, although still confined by the total volume limit.

Similar analysis has been done for some types of pressure-gain combustors (without evaporation or buffer gas). ${ }^{4}$ Recently, there has also been simplified gasdynamic analysis specifically for wave rotors, ${ }^{6}$ pulsed detonation engines, ${ }^{5}$ and work exchange devices. ${ }^{7}$ The purpose of this work is to isolate and generalize the purely thermodynamic considerations involved from the details of the gasdynamic and mechanical means utilized, while including the effects of buffer gas and liquid evaporation. Detailed analysis of pressure-gain devices such as wave rotors requires the application of compressible gas dynamics and/or combustion theory. As shown here, one can understand the possibilities of such devices from knowledge of thermodynamics only.

\section{Generalized Combustor Cycle}

Figure 2 is a schematic of a generalized pressure-gain or workproducing combustor. The steady-flow components upstream (for example, compressor) and downstream (for example, turbine) are considered to interact with many combustor units via large common reservoirs at states 2 and 3 .

The generalized cycle of charge, compression, evaporation, combustion, expansion, and discharge in one combustor unit are illustrated in Fig. 3. The processes are illustrated by a piston-cylinder device, but apply generally to any device in which the working gas

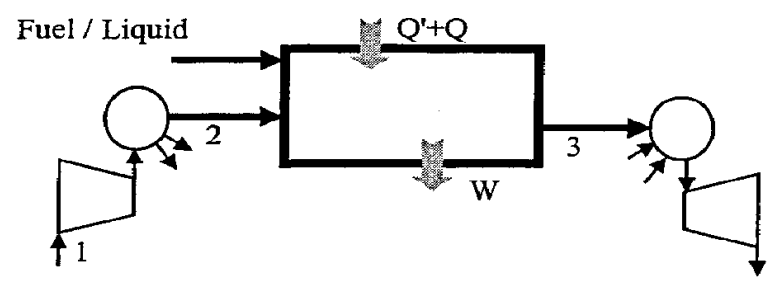

Fig. 2 Combustor system schematic.
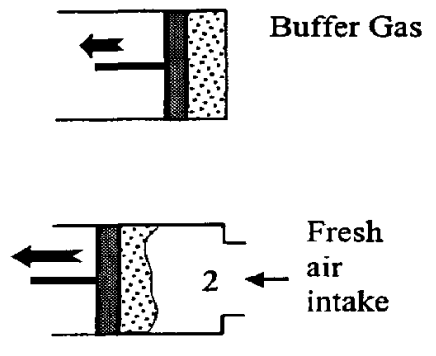

Pre-
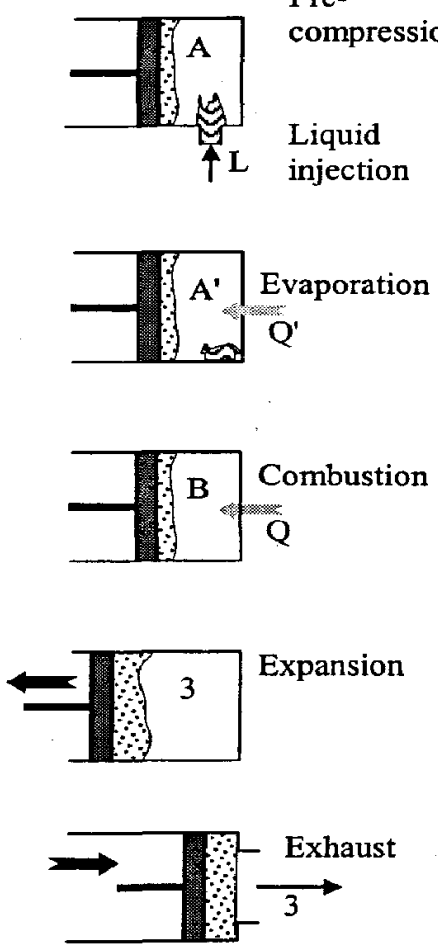

Fig. 3 Generalized combustion operating cycle. 
is subjected to the same processes. For instance, in a wave rotor the gas can enter and exhaust at opposite ends of a channel, with some previously burned and some unburned gas behaving effectively as a buffer gas during combustion. In pulse detonation engines and wave rotors some precompression might be provided by pressure waves in the chamber. From a thermodynamic point of view, what matters for work potential is the entropy change for a given heat release, which only depends on the pressure of an individual gas particle prior to combustion and evaporation and the volume change permitted to the particle during heat release. The ideal gain in work availability for a given initial state and heat release is thus influenced only by the degree of charge precompression and the amount and compressibility of any buffer gas in the combustion chamber.

The model does not include work exchange with gas that either does not enter the combustion chamber (for example, some ejector or bypass devices) or is pressurized after combustion (for example, some pulse detonation engines). However, this would be a straightforward extension of this study. Of special interest would be the addition of a generalized nonsteady work exchange device, whose performance limits are presented by Carlton. ${ }^{8}$ It is known that a detonation results in nearly the same entropy generation as a constant volume combustion process ${ }^{9}$; therefore, the current method applies approximately for a multitube PDE.

A pressure-gain combustion system does no net work by our definition, and the total work done on the piston must sum to zero over a cycle. Conversely, a work-producing combustion system has no net pressure gain, and the cycle must return the chamber to the inlet pressure before outflow begins. A confined combustion system could be constructed in which the benefit is obtained partially as work and partially as pressure gain, but the extension is left to the reader. Note that this model does not consider work-producing systems that continue heat addition during or after gas expansion to produce work (that is, "reheat" devices). The first law of thermodynamics is applied by matching the heat release to the overall temperature limits. The second law is implicit in the entropy generated at each subprocess as a function of system properties.

The gas in the chamber is considered to consist of the combustion gas and the buffer gas as shown in Fig. 3. Every particle of the working combustion gas is assumed to undergo the same process, so that the inflow and outflow states are each uniform. This is not true internally when combustion is accomplished by a propagating flame front, still less when the charge is fuel-stratified and not uniformly mixed, or significant kinetic energy is generated (detonation). For an ideal gas it can be shown that the pressure rise for a given heat addition to a mass of gas depends only on the accompanying volume change, irrespective of thermal nonuniformities. Such nonuniformities can still affect the gas dynamics of the device.

\section{Constant-Volume Combustion}

First consider the case of ideal constant-volume combustion with no buffer gas or liquid injection and with isentropic compression and expansion. The fresh charge is induced at pressure $p_{2}$ and optionally precompressed by the piston to pressure $p_{A}$. After combustion the burned gas is discharged at pressure $p_{3}$. This requires net positive flow work to be provided internally in the amount of $p_{3} v_{3}-p_{2} v_{2}=R\left(T_{3}-T_{2}\right)$, per unit mass, assuming an ideal gas with its gas constant unchanged by combustion. To provide this flow work, as well as any precompression work, the pressure $p_{B}$ developed by combustion must be sufficiently higher than the discharge pressure $p_{3}$; the internal expansion supplies this work. With reference to Fig. 4, states $A$ and $B$ are now distinguished from states 2 and 3 to account for optional compression and necessary expansion internal to the combustor. This nomenclature serves to emphasize the fact that we are here concerned with establishing the inflow-outflow relationships of the device, and when that is done its internal states will not be used for further analysis of the larger propulsion or power system. Typically, this larger system will be viewed as being similar to a Brayton cycle engine, but with the conventional steady heat addition device replaced by a pulsed combustor.

In the spirit of air-standard cycle analysis, the heat released by combustion will be treated as an external heat input, leaving the

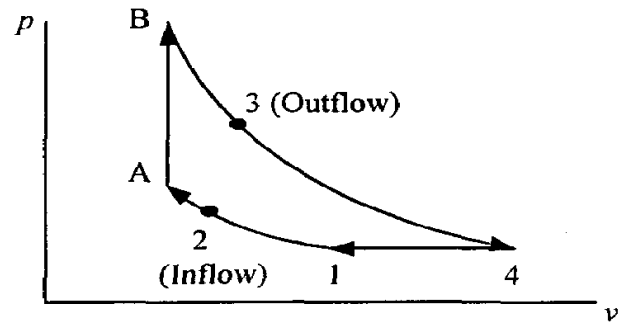

Fig. 4 Inflow and outflow states of constant volume combustor.

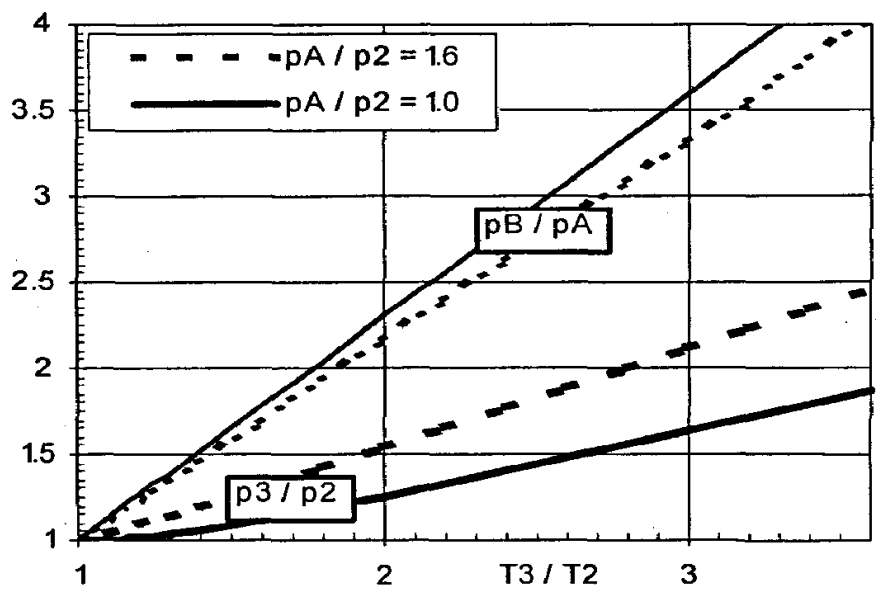

Fig. 5 Pressure gain for constant-volume combustion.

working gas composition unchanged. The net heat release $Q$ must equal the internal energy change of the trapped gas, mass $m$, during combustion from $\mathrm{A}$ to $\mathrm{B}$. We also require overall energy conservation for "steady-state" operation of a cyclic process in the combustion system with no accumulation. This requires that the net heat input $Q$ in a cycle also equal the sum of any net work done $W$ and the enthalpy change from two to three of the mass $m$. Therefore,

$$
Q=m c_{v}\left(T_{B}-T_{A}\right)=W+m c_{p}\left(T_{3}-T_{2}\right)
$$

It can be easily verified that the net work done on the piston summed over the individual steps of the cycle yields the same equality.

For pressure gain from state 2 to state 3 with no net work, $W=0$, so that from Eq. (1), and the ideal gas equation of state,

$$
T_{B}-T_{A}=\gamma\left(T_{3}-T_{2}\right)
$$

and

$$
p_{B} / p_{A}=T_{B} / T_{A}=1+\gamma\left(T_{3} / T_{2}-1\right)\left(T_{2} / T_{A}\right)
$$

and the pressure gain is

$$
\begin{aligned}
\frac{p_{3}}{p_{2}} & =\frac{p_{3}}{p_{B}} \frac{p_{B}}{p_{A}} \frac{p_{A}}{p_{2}}=\left(\frac{T_{3}}{T_{B}} \frac{T_{A}}{T_{2}}\right)^{\gamma /(\gamma-1)} \frac{T_{B}}{T_{A}} \\
& =\left(\frac{T_{3}}{T_{2}}\right)^{\gamma /(\gamma-1)}\left[1+\gamma\left(\frac{T_{3}}{T_{2}}-1\right)\left(\frac{p_{2}}{p_{A}}\right)^{(\gamma-1) / \gamma}\right]^{-1 /(\gamma-1)}
\end{aligned}
$$

The pressure gain $p_{3} / p_{2}$ and combustion pressure rise $p_{B} / p_{A}$ vary with the overall temperature ratio $T_{3} / T_{2}$, as presented in Fig. 5 . Note that the net pressure gain is considerably less that the constantvolume combustion pressure rise ratio. The effect of precompression by a pressure ratio of 1.6 , possible in the inlet process in a pulse detonation engine or wave rotor, is also shown by dashed lines.

For work production with no pressure gain, $p_{3}=p_{2}$, so that

$$
\frac{T_{B}}{T_{A}}=\frac{p_{B}}{p_{A}}=\frac{p_{B}}{p_{3}} \frac{p_{2}}{p_{A}}=\left(\frac{T_{B}}{T_{3}} \frac{T_{2}}{T_{A}}\right)^{\gamma /(\gamma-1)}
$$




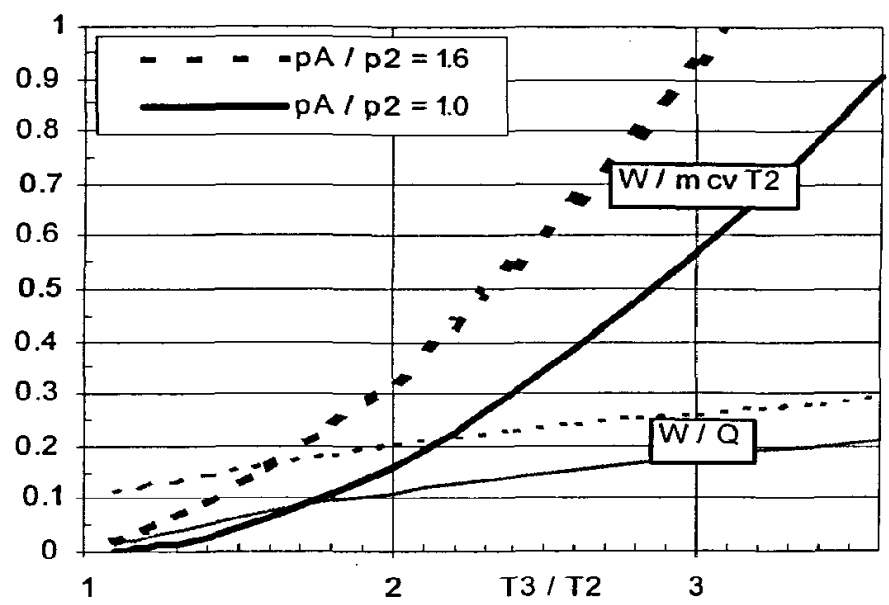

Fig. 6 Work production for constant-volume combustion.

Therefore,

$$
T_{B} / T_{A}=\left(T_{3} / T_{2}\right)^{\gamma}
$$

This equation should be contrasted with Eq. (1a). Further, from Eq. (1)

$$
\begin{aligned}
\frac{W}{Q} & =1-\gamma \frac{T_{3}-T_{2}}{T_{B}-T_{A}} \\
& =1-\gamma\left[\frac{T_{3} / T_{2}-1}{\left(T_{3} / T_{2}\right)^{\gamma}-1}\right]\left(\frac{p_{2}}{p_{A}}\right)^{(\gamma-1) / \gamma}
\end{aligned}
$$

The net work, nondimensionalized by $m c_{v} T_{2}$, and the "efficiency" $W / Q$ are plotted in Fig. 6. The efficiency is less meaningful if the work-producing combustor is operating in a combined cycle with a turbine. Note that $p_{B} / p_{A}$ is now independent of $p_{A} / p_{2}$ and has a simple form, and so it is not plotted.

\section{Effect of Buffer Gas and Irreversibilities}

The following derivation corresponds to Fig. 3, with buffer gas present, but no liquid injection. Further, the effect of losses during intake/precompression and expansion/discharge are represented by adiabatic efficiencies $\eta_{2 A}$ and $\eta_{3 B}$, respectively. The buffer gas is assumed to behave isentropically always. This might seem inconsistent, but the equivalent effect of irreversibilities can be assigned to the working gas, and it simplifies the model. In typical combustors the buffer gas is either replaced by newly combusted gas, or, if stagnant, it transfers entropy with heat to the working gas.

The pressure ratio of precompression is specified, and the temperature ratio is calculated:

$$
\frac{T_{A}}{T_{2}}=1+\frac{\left(p_{A} / p_{2}\right)^{(\gamma-1) / \gamma}-1}{\eta_{2 A}}
$$

Similarly, if the postcombustion temperature $T_{B}$ is determined, as discussed next, the pressure ratio of expansion is related to the temperature ratio:

$$
\frac{p_{3}}{p_{B}}=\left(1-\frac{1-T_{3} / T_{B}}{\eta_{3 B}}\right)^{\gamma /(\gamma-1)}
$$

To find $T_{B}$, we need to modify Eq. (1) to account for the buffer gas. The overall energy balance still applies:

$$
Q=W+m c_{p}\left(T_{3}-T_{2}\right)
$$

where $m$ is now the mass of working gas only.

Heat is released only during the combustion process from state $\mathrm{A}$ to $\mathrm{B}$ (for the buffer gas $r \mathrm{~A}$ to $r \mathrm{~B}$ ); the energy balance for the chamber is then:

$$
Q=m c_{v}\left(T_{B}-T_{\mathrm{A}}\right)+m_{r} c_{v}\left(T_{\mathrm{TB}}-T_{\mathrm{TA}}\right)
$$

with the total volume of gas kept constant:

$$
m v_{A}+m_{r} v_{\mathrm{rA}}=m v_{B}+m_{r} v_{\mathrm{rB}}
$$

Assuming that all gases have the same specific gas constant $R=p v / T$ and using Eq. (10),

$$
\begin{aligned}
\frac{p_{B}}{p_{A}} & =\frac{m T_{B}+m_{r} T_{\mathrm{rB}}}{m T_{A}+m_{r} T_{\mathrm{rA}}} \\
& =\frac{Q / c_{v}+m T_{A}+m_{r} T_{\mathrm{rA}}}{m T_{A}+m_{r} T_{\mathrm{rA}}}
\end{aligned}
$$

\section{Prescribed Buffer Gas Properties}

Suppose the initial state of the buffer gas, mass $m_{r}$, is prescribed by its temperature $T_{\mathrm{rA}}$. For the pressure-gain combustor $W=0, Q$ is found from Eq. (9), and Eq. (12) is used to find the combustion pressure ratio. As the pressure rises during combustion, the buffer gas is assumed to undergo an isentropic compression to a final temperature $T_{\mathrm{IB}}$ used in Eq. (10) to determine $T_{B}$ :

$$
T_{B}=T_{A}+Q / m c_{v}-\left(m_{r} / m\right) T_{\mathrm{rA}}\left[\left(p_{B} / p_{A}\right)^{(\gamma-1) / \gamma}-1\right]
$$

The pressure gain can now be calculated by determining the expansion pressure ratio from Eq. (8).

In the case of work production, the solution must be obtained by iteration. $p_{B} / p_{A}$ is guessed, perhaps using Eq. (12) for a seed value. The pressure gain is calculated from Eqs. (13) and (8), and the value of $p_{B} / p_{A}$ is adjusted until the pressure gain is unity. The work developed is then computed from Eq. (9).

\section{Burned Residual Gas Buffer}

It is often the case with pulse combustors and some wave rotor designs that nearly complete mixing of all gases occurs during the heat-release process, and the residual gas has the properties of the burned working gas at pressure $p_{B}$. It then compresses isentropically as a buffer gas over the next cycle of operation.

With this model and setting $T_{\mathrm{rB}}=T_{B}$, Eq. (12) is modified:

$$
\begin{gathered}
\frac{p_{A}}{p_{B}}=\frac{m T_{A}+m_{r} T_{\mathrm{rA}}}{m T_{B}+m_{r} T_{\mathrm{rB}}}=\mu \frac{T_{A}}{T_{B}}+(1-\mu)\left(\frac{p_{A}}{p_{B}}\right)^{(\gamma-1) / \gamma} \\
T_{B}=\mu T_{A}\left[\frac{p_{A}}{p_{B}}-(1-\mu)\left(\frac{p_{A}}{p_{B}}\right)^{(\gamma-1) / \gamma}\right]^{-1}
\end{gathered}
$$

where $\mu=m /\left(m+m_{r}\right)$. Also, from Eq. (10),

$$
Q=\left(m+m_{r}\right) c_{\nu} T_{B}\left(1-p_{A} / p_{B}\right)
$$

Closed-form solutions are not obtained for either pressure-gain or work-producing combustors. One approach to iteration is to guess $p_{A} / p_{B}$ and use Eq. (14) to calculate the corresponding $T_{B}$. For the case of pressure gain, use Eq. (15) to calculate the corresponding $Q$, check it against the correct value from $\mathrm{Eq}$. (9), and reiterate until $W=0$. For the case of work production, also guess $p_{A} / p_{B}$ and use the corresponding $T_{B}$ to calculate $p_{3} / p_{B}$ from Eq. (8). Then check the corresponding pressure gain, and reiterate until it is unity.

\section{Results}

Particular numerical methods for iteration are not described here. The following solutions were obtained by setting up a spreadsheet software program to perform the computations using an internal iterative method. The ideal pressure gain computed for constantvolume combustion with a precompression ratio of 1.6 is reproduced in Fig. 7 (heavy full line). When a buffer gas with the properties of fresh inlet air is present at a mass ratio of $40: 60$ to the combustible working gas $(\mu=0.6)$, the pressure gain is lower (heavy dashed line). When the same mass of buffer gas has the properties of burned 


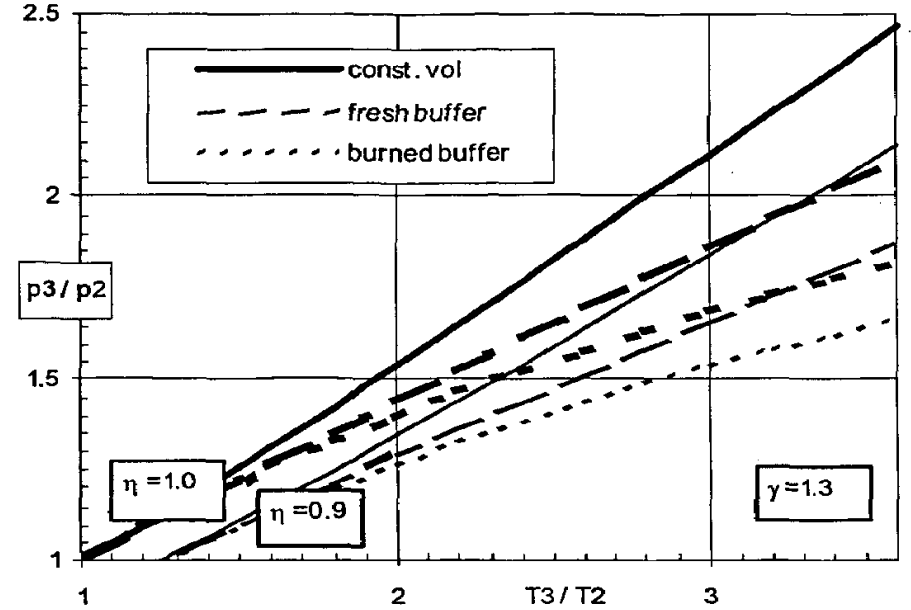

Fig. 7 Pressure gain with buffer gas and losses.

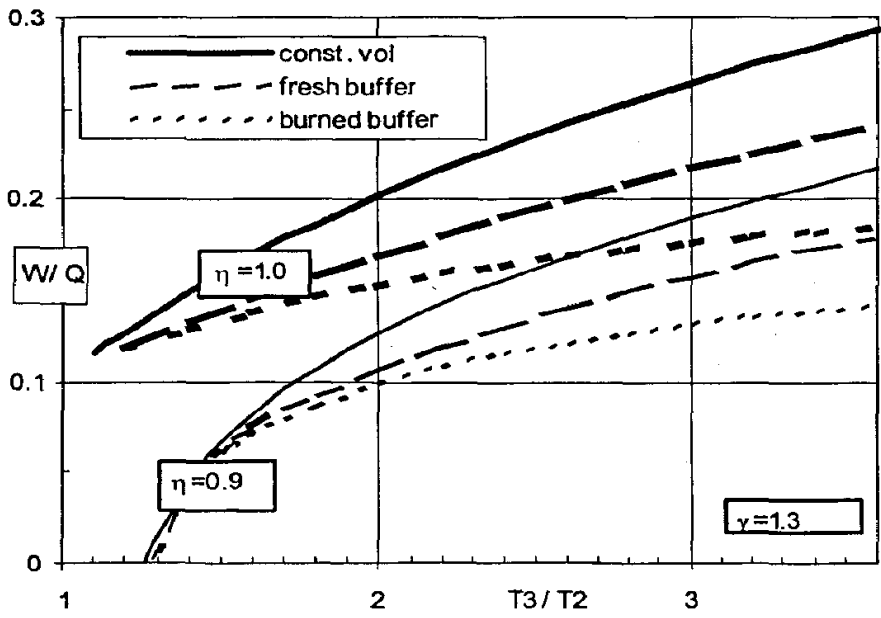

Fig. 8 Work production with buffer gas and losses.

gas, the pressure gain is even lower (heavy dotted line). Corresponding plots (lighter lines) are also shown for the constant-volume and buffered cases when the compression and expansion processes have adiabatic efficiency, $\eta=\eta_{2 A}=\eta_{3 B}=0.9$. For small $T_{3} / T_{2}$ the inefficiencies can result in a net pressure loss. Most practical engines, even at low load conditions, will operate at a combustion temperature ratio that promises significant performance improvement with pressure-gain combustion.

In Fig. 8 are shown the corresponding plots for nondimensional work production efficiency. The presence of buffer gas and irreversibilities cause the work production to deteriorate with trends similar to the pressure gain. Similar trends would also be seen for the mass-specific work, not shown here.

\section{Applications}

The preceding general results can be applied to many examples of pulsed combustion devices to estimate the thermodynamic limit performance. Three cases are discussed next, all of which incorporate the basic Humphrey cycle, but within different propulsion or power systems. The combustion process in each of these cases could potentially be implemented in a wave rotor or in similar valved mechanical devices. Figure 9 is an illustration of a simple combustion wave rotor, consisting of a large number of passages on the periphery of a rotor. As it rotates, a fuel-air mixture from a partial annular inflow duct enters each passage, is brought to rest through a compression (shock) wave, is ignited and burns in a closed volume, and is expanded and accelerated out of each passage into an exhaust duct. The combustion process might be a deflagration or detonation. With the channels straight and axial as shown, no mechanical work

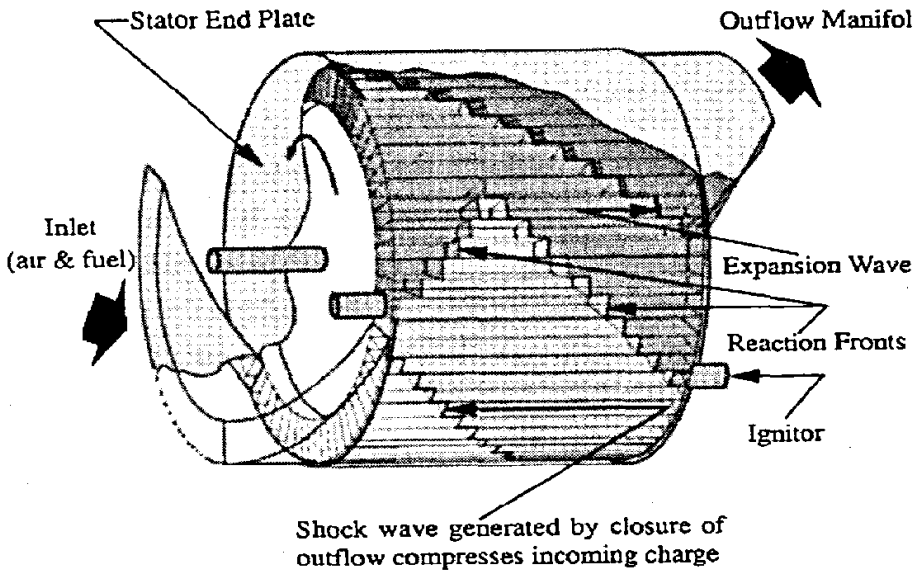

Fig. 9 Illustration of a combustion wave rotor.

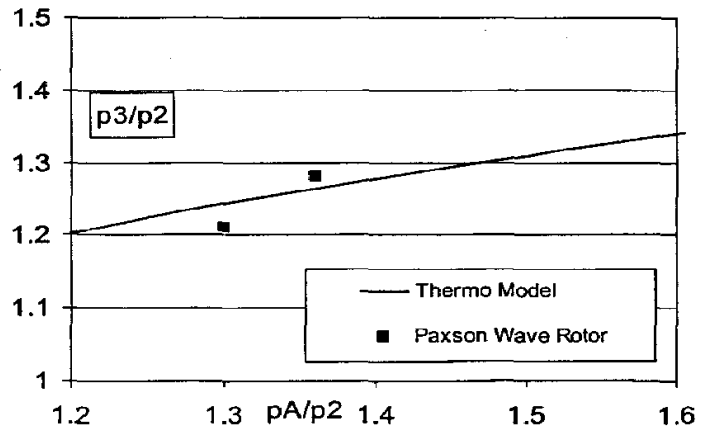

Fig. 10 Wave rotor simulation comparison.

is extracted, and a pressure gain will be realized between inlet and outlet. The pressurized flow can be utilized in a turbine or in a nozzle for direct thrust. On the other hand, if the channels are slanted and/or curved the wave rotor can act as a wave turbine that extracts work by transfer of angular momentum.

\section{Naturally Aspirated Power Unit Performance}

The maximum performance of a wave turbine or other workproducing combustion device with atmospheric inlet and outlet conditions can be estimated from the preceding procedure. Constantvolume combustion of an uncompressed stoichiometric propane-air mixture at $520^{\circ} \mathrm{R}$ results in pressure and temperature rising by a factor of eight, approximately. For $T_{B} / T_{A}=8$, without precompression and with no losses, $W=1.9 c_{v} T_{2}$, or about $310 \mathrm{HP}-\mathrm{s} / 1 \mathrm{~b}_{\mathrm{m}}$. The maximum efficiency is $27 \%$. This ideal work limit can be exceeded only by "reheat" devices. Consider now a wave turbine with partial purging of the combustion chamber and efficient aspiration that yields a wave precompression ratio of 1.6 . We assume residual burned buffer gas with $40 \%$ mass fraction and adiabatic efficiency of 0.9 for compression and expansion. The maximum work production for this more realistic system is $220 \mathrm{HP}-\mathrm{s} / \mathrm{lb}_{\mathrm{m}}$, and the maximum efficiency is $16 \%$. The reader can apply the same procedurc for more compressed charge devices, such as piston engines, for comparison.

\section{Contoured-Passage Wave Rotor}

This thermodynamic modeling approach can be compared with detailed simulations of advanced wave rotor performance. Variation of passage height profile in a wave rotor design affects pressure gain, apparently through differences in precompression ratio and, to a lesser extent, expansion efficiency. Figure 10 is a plot of pressure gain against precompression ratio for a combustor with burned residual gas (solid line, $\gamma=1.353, \mu=0.65, \eta_{2 A}=\eta_{3 B}=0.9$ ). A couple of wave rotor gas dynamic simulations, ${ }^{10}$ for $T_{3} / T_{2}=2.21$, show reasonably good agreement for pressure gain (square symbols) with this model, for which the process efficiencies and buffer gas mass 
just given were roughly estimated from simulation data. Although the wave rotor simulations were for a four-port pressure-gain device with additional wave processes, it was equivalent in inlet and outlet states to a combustion wave rotor. ${ }^{11}$ Similar detailed simulations of pulse detonation cycles are available; however, comparison with the thermodynamic model is complicated by the difficulty of estimating an expansion efficiency, which includes the effect of eliminating severe nonhomogeneity in the exhaust gas for which a uniform state is to be compared.

\section{Pressure-Gain Ramjet}

This application considers the benefit of pressure-gain combustion for a ramjet engine. Consider first the ideal steady-flow conventional ramjet with no losses and perfect gas behavior, which operates on the Brayton cycle. We assume $\gamma=1.25$, the temperature rise by stoichiometric combustion of hydrocarbon fuel is constant at $3000^{\circ} \mathrm{R}$, and the high-altitude ambient temperature is $400^{\circ} \mathrm{R}$. The solid line in Fig. 11 is the specific impulse $I_{s p}$ of the ideal ramjet with no intake or combustor losses and with complete nozzle expansion. The impulse calculated at each flight Mach number assumes the appropriate inlet and nozzle geometry at that speed for an ideal stoichiometric hydrocarbon ramjet. As is well known, $I_{\mathrm{sp}}$ goes from zero at static conditions to a maximum (about $1400 \mathrm{~s}$ at about $M=4$ ) and then falls gradually as speed increases. This ideal ramjet has zero velocity at combustion, requiring infinite frontal area. A real ramjet suffers a penalty to keep area limited.

Next, the specific impulse is calculated for a similarly idealized pressure-gain combustion ramjet. This result (dashed line, Fig. 11) is based on the theoretical pressure rise if the combustion process is partially confined such as in a wave rotor or valved combustor. The pressure rise depends only on three parameters: the temperature rise, the presence of gas in the combustor that does not undergo combustion, and any precompression such as caused by wave action during intake into the combustor. It is assumed conservatively, based on typical wave rotor gasdynamics, that $50 \%$ of the combustion chamber is refilled and burned in each cycle of the unsteady combustor, as a mass fraction of the total confined gas, and that the residual gas has the properties of unburned gas. We also assume a slight precompression pressure ratio of 1.2. It was found that variations in the filled fraction, residual gas properties, and precompression ratio up to 2.0 do not change the curve significantly. Given ideal ram pressure for each flight speed at the valved combustor inlet, the pressure at the combustor exit is determined with the preceding parameters. The thrust is then calculated assuming an isentropic expansion of the combustor outflow gas from the boosted pressure to atmospheric conditions. More details of this calculation procedure are given in Ref. 12.

In essence, this shows that 1) the performance of a pressuregain ramjet is always better than a simple ramjet and 2) from static operation up to where the maximum $I_{\text {sp }}$ of a simple ramjet occurs (about Mach 4) the pressure-gain ramjet has a roughly constant $I_{\mathrm{sp}}$ that is always higher than this maximum. A PDE ramjet is expected to have very similar performance.

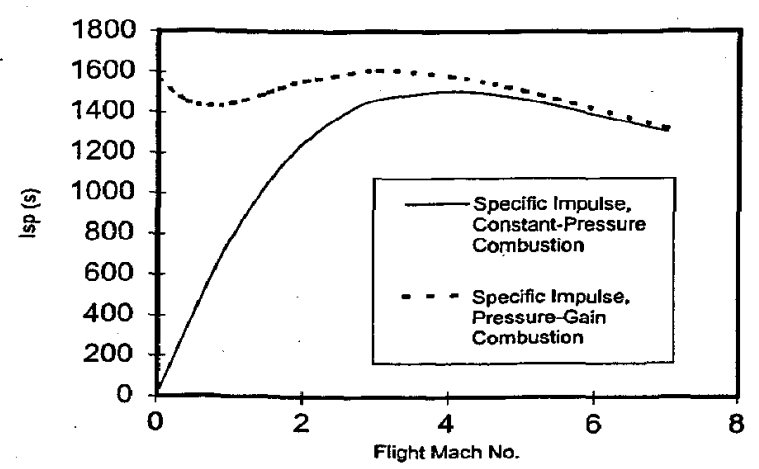

Fig. 11 Pressure-gain combustion for a stoichiometric hydrocarbon ramjet.

\section{Evaporation}

In many combustion devices liquid fuel is injected and evaporated before combustion with the help of atomization during injection and heat from combustion. Simplified models of combustion thermodynamics, such as used in the prior sections, do not account for the effect of this evaporation. "Air-standard" heat-addition models also do not account for many other effects of real combustion, including changes in gas specific heat and molar density, as a result of composition change (including dissociation) and large temperature change. Here we examine only the effect of liquid evaporation, insofar as it affects the gas molar density and pressure. The change in number of moles as a result of chemical conversion can be appended to the evaporative increase, if desired, but will not be meaningful unless other real-gas effects are also included. The evaporative effect applies to fuel or propellant injection and any other liquid injection, such as demineralized water in "humidified" turbine engines ${ }^{13}$ to enhance engine power or control emissions.

It is assumed that the relative amount of liquid injected is sufficiently small that the average gas molecular weight and specific heats do not change as a result of the evaporated vapor. It is also assumed that the vapor is dilute and behaves as an ideal gas. For simplicity, liquid evaporation is considered only for the constantvolume case with no buffer gas.

Referring to Fig. 3, we now distinguish between states $\mathrm{A}$ and $\mathrm{A}^{\prime}$, before and after the evaporation process, which involves a heat input $Q^{\prime}$. The heat input is defined to be the heat required to evaporate the liquid such that the final temperature of the mixture is the same as the initial temperature of the air $T_{A}$. With constant volume and temperature, the pressure ratio is equal to the molar ratio of $A^{\prime}$ to $A$.

$$
p_{A^{\prime}} / p_{A}=n_{A^{\prime}} / n_{A}
$$

Energy conservation for the cycle now requires

$$
Q+Q^{\prime}=W+m_{2} c_{p}\left(T_{3}-T_{2}\right)+m_{L}\left(h_{3}-h_{L}\right)
$$

By definition,

$$
Q^{\prime}=m_{L}\left(u_{A^{\prime}}-\dot{u}_{L}\right)
$$

and we assume that the specific volume of the liquid is negligible, so that $h_{L} \approx u_{L}$.

Also, for combustion,

$$
Q=\left(m_{2}+m_{L}\right) c_{v}\left(T_{B}-T_{A}\right)
$$

Paralleling the derivation of pressure gain in Eqs. (2) and (3), for constant-volume combustion with isentropic compression and expansion processes

$p_{B} / p_{A^{\prime}}=T_{B} / T_{A^{\prime}}=1-\mu_{L}+\gamma\left(T_{3} / T_{2}-1+\mu_{L}\right)\left(T_{2} / T_{A}\right)$

where $\mu_{L}=m_{L} /\left(m_{2}+m_{L}\right)$, and the pressure gain is

$$
\begin{aligned}
\frac{p_{3}}{p_{2}}= & \frac{p_{3}}{p_{B}} \frac{p_{B}}{p_{A^{\prime}}} \frac{p_{A^{\prime}}}{p_{A}} \frac{p_{A}}{p_{2}} \\
= & \left(\frac{T_{3}}{T_{B}} \frac{T_{A}}{T_{2}}\right)^{\gamma /(\gamma-1)} \frac{T_{B}}{T_{A^{\prime}}} \frac{n_{A^{\prime}}}{n_{A}} \\
= & \left(\frac{T_{3}}{T_{2}}\right)^{\gamma /(\gamma-1)} \frac{n_{A^{\prime}}}{n_{A}} \\
& \times\left[1-\mu_{L}+\gamma\left(\frac{T_{3}}{T_{2}}-1+\mu_{L}\right)\left(\frac{p_{2}}{p_{A}}\right)^{(\gamma-1) / \gamma}\right]^{-1 /(\gamma-1)}
\end{aligned}
$$

Similarly, the nondimensional work production without pressure gain can be shown to be 


$$
\begin{aligned}
\frac{W}{m_{2} c_{\nu} T_{2}} & =\left\{\left(\frac{T_{3}}{T_{2}}\right)^{\gamma}\left(\frac{n_{A^{\prime}}}{n_{A}}\right)^{\gamma-1}-1+\mu_{L}\right\}\left(\frac{p_{A}}{p_{2}}\right)^{(\gamma-1) / \gamma} \\
& -\gamma\left\{\frac{T_{3}}{T_{2}}-1+\mu_{L}\right\}
\end{aligned}
$$

For combustion with evaporation, the heat added cannot be expressed only in terms of the sensible energies of the inflow and outflow states. Instead, the heat of evaporation must be obtained from physical data for the particular liquid. Therefore, an efficiency similar to Eq. (6) is not calculated as it will be meaningless without including the heat of evaporation. As expected, the work production without liquid injection is recovered from Eq. (22) after some algebraic manipulation by setting $u_{L}=0$ and $n_{A^{\prime}} / n_{A}=1$, and comparing with Eq. (6) while setting $Q=m c_{p}\left(T_{3}-T_{2}\right)$ there. The effect of evaporation will be illustrated for cases of fuel evaporation in the closed combustion chamber and water injection. The composition of the gas after mixing of hydrocarbon or hydrogen fuel, water, and air is represented as

$$
\phi \mathrm{C}_{x} \mathrm{H}_{y}+(x+y / 4)\left(\mathrm{O}_{2}+3.76 \mathrm{~N}_{2}\right)+\alpha \mathrm{H}_{2} \mathrm{O}
$$

\section{Fuel Evaporation}

Consider the case of stoichiometric $(\phi=1)$ hydrogen-air combustion with initial temperature such that $T_{3} / T_{2}=6.0$. Without precompression and assuming $\gamma=1.2$, constant-volume combustion of a premixed charge will result in an ideal net pressure gain of 2.78. Instead of premixing, injection of liquid hydrogen ( $\left.\mu_{L}=0.144, n_{A^{\prime}} / n_{A}=1.42\right)$ after closing the combustion chamber will yield a pressure gain of 3.06 or about $10 \%$ better. Similar calculations were done for typical hydrocarbon fuels burning in air at lean overall fuel-air ratios typical of gas turbines such that $T_{3} / T_{2} \approx 2$. The pressure gain increases by $1 \%$ or less as a result of the very small molar density of fuel. When the relative amount of liquid is large, such as in a pulsed combustion rocket chamber with liquid fuel and oxidizer propellants, the evaporation effect is likely to be substantial, but is not considered in this paper.

\section{Water Injection}

An interesting example is that of liquid water injection into the chamber. At a typical level of humidification for powergeneration gas turbines, $\mu_{L}=0.15, n_{A^{\prime}} / n_{A}=1.25$, the pressure gain is increased from 1.53 to 1.84 , a $20 \%$ improvement (assuming $\gamma=1.3, T_{3} / T_{2}=2$, precompression ratio of 1.6 ). The case of a work-producing device with no pressure gain is even more impressive. For the same conditions the nondimensional specific work increases from 1.1 to 1.64 units, a $50 \%$ increase. This benefit is in addition to the turbine output boost from humidification at constant pressure. Humidification can be practical only for temporary power boost and environmental compliance under high ambienttemperature conditions. A work-producing combustion device allows the limited quantity of water injected to have maximum impact. The pressure gain for constant-volume combustion, without

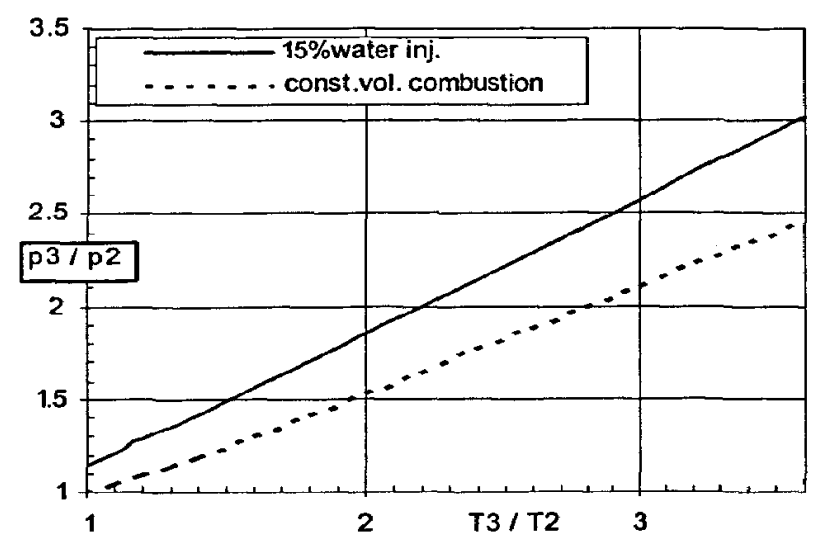

Fig. 12 Pressure-gain enhancement by evaporation.

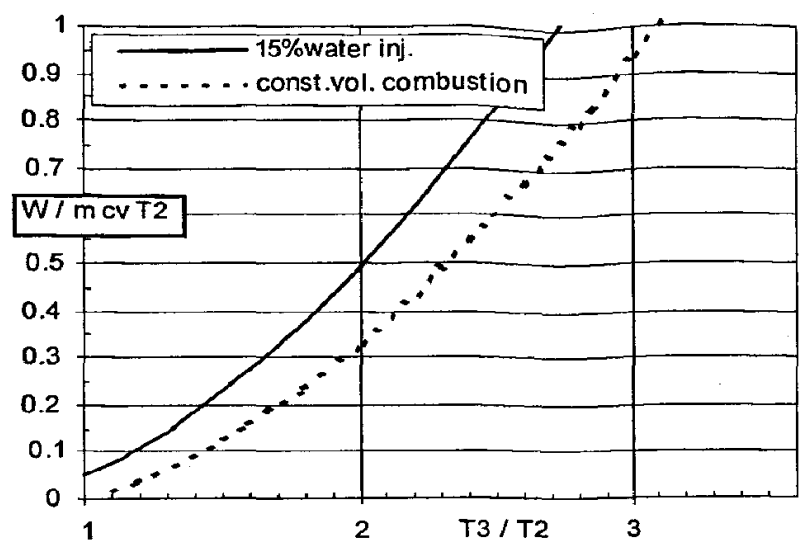

Fig. 13 Work enhancement by evaporation.

and with water injection with a mass fraction of $15 \%$, is plotted in Fig. 12 for a range of temperature ratios. Figure 13 is a corresponding plot of work production.

\section{Conclusions}

A thermodynamic model has been developed to analyze pressure gain and work production in systems where combustion and evaporation occur in air in a closed volume. The ideal case of a constant volume combustor allows simple algebraic expressions to be developed for the maximum pressure gain or work production that is thermodynamically possible.

The effects of a residual buffer gas and friction losses are included in a more detailed model. Iterative calculations are usually needed to modify the thermodynamic limits for these effects. Examples of devices are provided for which specific performance limits can be derived. A comparison is made with published gas dynamic computations for a wave rotor.

The effect of fuel vaporization or other liquid evaporation in the combustion chamber is considered. Fuel vaporization has a significant effect only for low-molecular weight fuel (hydrogen). Substantial effects on pressure gain or work production results when liquid water is injected into a confined combustor. The practical feasibility of exploiting this work potential is yet unknown, and may depend on any other constraints placed on the system.

\section{References}

${ }^{1}$ Bussing, T., and Pappas, G., "An Introduction to Pulse Detonation Engines," AIAA Paper 94-0263, Jan. 1994.

${ }^{2}$ Moran M. J., and Shapiro, H. N., Fundamentals of Engineering Thermodynamics, 4th ed., Wiley, 2000 , pp. 425-450.

${ }^{3}$ Welch, G. E., "Wave Engine Topping Cycle Assessment," AIAA Paper 97-0707, Jan. 1997.

${ }^{4}$ Kentfield, J. A. C., and O'Blenes, M., "Methods of Achieving a Combustion-Driven Pressure Gain in Gas Turbines," Transactions of the ASME, Vol. 110, No. 4, 1988, p. 704-711.

${ }^{5}$ Welch, G. E., and Paxson, D. E., "Wave Turbine Analysis Tool Development," AIAA-98-3402, July 1998; also NASA TM-1998-208485, July 1998, and ARL-TR-174, Army Research Laboratory, Cleveland, OH, July 1998.

${ }^{6}$ Welch, G. E., "Macroscopic Balance Model for Wave Rotors,"' Journal of Propulsion and Power, Vol. 13, No. 4, 1997, pp. 508-516.

${ }^{7}$ Kentfield, J. A. C, "The Fundamentals of Idealized Air-Breathing PulseDetonation Engines," Journal Propulsion and Power, Vol. 18, No. 1, 2002, pp. 77-83; AIAA Paper 2000-3469, July 2000.

${ }^{8}$ Carlton, R. A., "Fundamental Limits of Performance of Nonsteady Work Exchange Devices," Master's Thesis, Dept. of Mechanical Engineering, Univ. of Florida, Gainesville, Dec. 1994.

${ }^{9}$ Kuo, K. K., Principles of Combustion, Wiley, New York, 1986, p. 242.

${ }^{10}$ Paxson, D. E., and Lindau, J. W., "Numerical Assessment of Four-Port, Through-Flow Wave Rotor Cycles with Passage Height Variation," AIAA Paper 97-3143, July 1997; also NASA TM 107490, July 1997.

${ }^{11}$ Nalim, M. R., "Assessment of Combustion Modes for Internal Combustion Wave Rotors," Journal of Engineering for Gas Turbines and Power, Vol. 121, No. 2, 1999, pp. 265-271.

${ }^{12}$ Nalim, M. R., and Jules, K., "Pulse Combustion and Wave Rotors for High Speed Propulsion Engines," AIAA Paper 98-1614, April 1998.

${ }^{3}$ Nakhamkin, M., Swensen, E. C., Wilson, J. M., Gaul, G., and Polsky, M., "The Cascaded Humidified Advanced Turbine," Journal of Engineering for Gas Turbines and Power, Vol. 118, No. 3, 1996, pp. 565-571. 